\title{
Ligadura de fuga venosa dorsal profunda en pene: Revisión de la literatura y técnica quirúrgica paso a paso
}

\section{Penile Deep Dorsal Venous Leakage: Review of Literature and Surgical Technique Step by Step}

Juan Fernando Uribe Arcila1(1) ${ }^{1}$ Departamento de Urología, Hospital Pablo Tobón Uribe, Medellín,
Colombia

Urol Colomb 2020;29:48-57. Address for correspondence Juan Fernando Uribe Arcila, MD,
Departamento de Urología, Hospital Pablo Tobón Uribe,
Medellín 050026, Colombia (e-mail: medicinasexual@une.net.co).

\section{Resumen \\ Palabras clave \\ - pene \\ - disfunción eréctil \\ - disfunción eréctil primaria \\ - disfunción veno- oclusiva}

La disfunción venoclusiva peneana (VOD), está siendo reconocida como una causa importante de disfunción eréctil en especial en pacientes jóvenes. Recientemente se han sugerido fisiopatologías novedosas y se han mejorado notablemente los métodos diagnósticos, lo que genera un campo en redescubrimiento con nuevos enfoques de tratamiento disponibles. La ligadura de fuga venosa dorsal ha tenido dificultades a través de la historia para ser estandarizada. El artículo enfatiza que el diagnóstico adecuado es la base del éxito en esa patología, para cuya resolución presentamos una técnica quirúrgica asequible, aún en sitios con limitaciones tecnológicas, reproducible y con buenos resultados a corto y mediano plazo. Cuando se sospecha de una fuga venosa dorsal profunda que cause alteraciones hemodinámicas con un cierto tipo de disfunción eréctil, con penes que en erección alternan en estados de duro-blando, se configura un síndrome especial, con hallazgos clínicos y ecográficos típicos. Aunque existen algunas opciones médicas de tratamiento, se propone la corrección quirúrgica de la fuga venosa como el método ideal de tratamiento definitivo.

Venoclusive dysfunction (VOD) is being recognized as an important cause of erectile dysfunction, especially in young patients. Novel pathophysiology has been suggested and diagnostic methods have been significantly improved, generating a field in rediscovery with new treatment approaches available. Ligation of dorsal venous leak has had difficulties throughout history to be standardized. The article emphasizes that the proper diagnosis is the basis of success in this pathology, for whose resolution we present an affordable surgical technique, even in places with technological limitations, reproducible and with good results in the short and medium term. When a deep dorsal venous leak is suspected that causes hemodynamic alterations and a certain type of erectile dysfunction, with hard-flaccid penis, a special syndrome is configured, with typical clinical and ultrasound findings. Although there are some medical treatment options, it proposes the surgical correction of venous leakage as the ideal method of definitive treatment. received

June 23, 2019

accepted

September 18, 2019
DOI https://doi.org/

10.1055/s-0039-1698815.

ISSN 0120-789X.

e ISSN 2027-0119.
Copyright (c) 2020, Sociedad Colombiana License terms de Urología. Publicado por Thieme Revinter Publicações Ltda., Rio de Janeiro, Brazil. Todos los derechos reservados. 


\section{Introducción}

Los mecanismos de la erección humana son una compleja e intrincada red donde intervienen factores centrales, hormonales, neurológicos, arteriales y venosos. El peso de cada uno de esos elementos es variable y a menudo desconocido, sin embargo, la disfunción venoclusiva peneana (VOD por sus siglas en inglés), está siendo reconocida, casi redescubierta, como una causa importante de disfunción eréctil en especial en pacientes jóvenes. ${ }^{1}$ En años recientes, se ha sugerido una asociación entre la ultra estructura del pene, especialmente en la arquitectura de la túnica albugínea y la presencia de fugas venosas, lo que hace de esa etiología, un campo que apenas comienza a estudiarse adecuadamente, con nuevos enfoques de tratamiento disponibles y buscando resolver adecuadamente los múltiples interrogantes que surgen con los estudios. ${ }^{2}$ La clave de un tratamiento exitoso debe estar basada en la cuidadosa selección de los pacientes, por lo que el motivo principal de este nuevo pico de interés por las fugas venosas, se fundamenta en el arribo de mejores métodos para refinar el diagnóstico como el ultrasonido con imágenes de alta definición, la cavernosografía TAC o la Resonancia magnética nuclear peneana, que permitirán una mejor correlación con la clínica y una mejor escogencia de los que pueden beneficiarse de un tratamiento quirúrgico.

\section{Historia de la Cirugía de Fugas Venosas}

Más de 100 años de historia no han sido suficientes para que el tema de las fugas venosas madure y se decante adecuadamente. La cirugía de fugas venosas data desde el siglo XIX, con Raymond and Duncan en $1895^{3}$ y a lo largo de la historia el entusiasmo por esas técnicas ha tenido marcadas variaciones. La cirugía de ligadura está reportada desde comienzos del siglo XX por Wooten, ${ }^{3}$ como la cura del pene átono $\mathrm{y}$ fue posteriormente mencionada por diferentes autores hasta su florecimiento en la década de los 80 donde se publicaron múltiples estudios sobre el tema, incluyendo a Wespes/Shulman ${ }^{4,5}$ Lazzarini, ${ }^{6}$ Shabsigh $^{7}$ y Lue. $^{8}$ Esa revisión menciona adicionalmente otros artículos de referencia sobre el tema como Florez/Mulhall, ${ }^{9}$ Cayan $^{10,11}$, Shultheis, ${ }^{12}$ Lukkarinen, ${ }^{13}$ Basar, ${ }^{14}$ Al Assal, ${ }^{15}$ Sasso, ${ }^{16}$ Popken ${ }^{17}$ y Rao/Donatucci, ${ }^{18}$ con sus respectivos resultados altamente variables. El núcleo del problema fue mencionado en el artículo de Trost/Munarrriz, ${ }^{19}$ quienes se quejan de la dificultad de encontrar literatura relevante acerca de la cirugía venosa del pene y que con la información disponible no se pueden realizar análisis que permitan la estandarización en la selección de los pacientes, test hemodinámicos, técnica quirúrgica, seguimiento $\mathrm{y}$ resultados definitivos. Un renacer del mecanismo fisiopatológico y la propuesta de diferentes técnicas quirúrgicas para afrontarlo, ocurrió en los últimos años con las publicaciones del urólogo coreano Geng L. $\mathrm{Hsu}^{20-22}$ también reseñadas en este artículo. (Ver - Tabla 1)

\section{Fisiopatología de las Fugas Venosas Peneanas}

Existen dos tipos de fugas venosas dorsales posibles: Las primarias o congénitas y las secundarias. Las segundas que forman parte de enfermedades que cursan con fugas venosas adquiridas afectando diferentes topografías del pene, y con etiologías diferentes que no forman parte de esta revisión. Desde hace más de un siglo, existe una gran controversia en la fisiopatología de las fugas venosas dorsales congénitas o primarias que puedan afectar la hemodinámica del pene y producir una alteración persistente de la calidad de la erección no existiendo una teoría única. ${ }^{23}$ La primera opción, es una condición intrínseca de la vena semejante a la que ocurre en los pacientes con varicocele u otro tipo de disfunciones venosas periféricas, que la hace insuficiente a largo plazo. Otra opción, es la alteración del tejido circundante, en especial de la túnica albugínea (TA) como origen de las fugas; según esa teoría, la posición del plexo venular subtunical y las venas emisarias perforantes que pasan a través de él entre los sinusoides y la TA, permiten su compresión y oclusión a medida que el músculo liso y las sinusoides se relajan y se expanden por entrada de un gran flujo sanguíneo en contra el tejido fibroelástico de la TA durante la tumescencia, lo que atrapa la sangre dentro del pene, teniendo en cuenta que la TA, compuesta principalmente de fibras de colágeno, es un tubo relativamente poco complaciente que rodea el tejido corporal del pene. ${ }^{2}$ Cuando el colágeno de la TA se encuentra degenerado y atrófico, probablemente conduce a una subluxación que no logra una adecuada compresión en ese tubo rígido; la pérdida de esa función venoclusiva, conduce a una fuga de sangre del pene con la subsecuente disfunción eréctil en esos pacientes. Sin embargo, debe considerarse que si la calidad de la túnica albuginea (atrofia o fibrosis), es el factor principal en la fisiopatología de las fugas venosas, es también la razón por la que a menudo la cirugía es inefectiva. ${ }^{24,25}$

\section{Perfil del Paciente con Síndrome de Fuga Venosa Dorsal Profunda}

La guía de la AUA sobre el manejo de la disfunción eréctil ${ }^{26}$ y la práctica estándar de ISSM en medicina sexual, no recomiendan cirugías realizadas para limitar la fuga venosa del pene. Sin embargo, publicaciones recientes sugieren que la cirugía venosa del pene para la fuga venosa dorsal profunda congénita en una población altamente seleccionada de hombres jóvenes, puede producir excelentes resultados a largo plazo y satisfacción del paciente al constituirse en una causa primaria curable de la disfunción eréctil en pacientes jóvenes. ${ }^{4}$ La disfunción eréctil primara (DEP) o la disfunción eréctil que empieza antes de los 40 años o secundaria no estándar (DESNE), implican para el clínico un reto diagnóstico ante la posibilidad de que ese tipo de pacientes puedan ser sometidos a procedimientos quirúrgicos curativos de la disfunción. Todos los pacientes jóvenes con disfunción eréctil, sobre todo con patrón de perdida fácil o con un patrón 
Tabla 1 Resultados de diferentes autores en ligadura o embolización de venas dorsales

\begin{tabular}{|c|c|c|c|c|c|c|}
\hline Autor & $\mathrm{n}$ & Edad & Técnica & $\begin{array}{l}\text { Tiempo de } \\
\text { seguimiento } \\
\text { (meses) }\end{array}$ & $\begin{array}{l}\text { Método de } \\
\text { seguimiento }\end{array}$ & Resultados \\
\hline Sasso & 23 & $\begin{array}{l}20-50 \\
(419)\end{array}$ & $\begin{array}{l}\text { Ligadura de superficial, } \\
\text { profunda, circunflejas } \\
\text { y emisarias }\end{array}$ & 12 & NS & $\begin{array}{l}\text { Erección espontanea } \\
\text { en } 74 \% \text { y 55\% largo } \\
\text { término }\end{array}$ \\
\hline Popken & 122 & $\begin{array}{l}19-78 \\
(49)\end{array}$ & $\begin{array}{l}\text { Ligadura de superficial, } \\
\text { profunda, circunflejas }\end{array}$ & 70 & Cuestionario & $\begin{array}{l}\text { Erección espontanea } \\
\text { en } 14 \%\end{array}$ \\
\hline Al Assal & 325 & $\begin{array}{l}18-62 \\
(45)\end{array}$ & NS & $1-13$ & NS & $\begin{array}{l}\text { Curados } 76 \text { ptes }<40 a \text {, } \\
58 \text { ptes }>40 a\end{array}$ \\
\hline Lukkarinen & 21 & $\mathrm{NA}$ & NS & $\geq 12$ & $\begin{array}{l}\text { Reporte del } \\
\text { paciente }\end{array}$ & $\begin{array}{l}\text { Buena en } 29 \% \\
\text { IIC en } 52 \%\end{array}$ \\
\hline Basar & 26 & NA & NS & 25 & NS & $\begin{array}{l}\text { Erección completa } \\
\text { en } 15 \% \text {, parcial en } 23 \%\end{array}$ \\
\hline Shultheiss & 147 & NA & NS & NA & Cuestionario & $\begin{array}{l}\text { Erección espontanea } \\
\text { en } 11,2 \%\end{array}$ \\
\hline $\begin{array}{l}\text { Fernandez } \\
\text { Arjona }\end{array}$ & 23 & 63 & Ballón transfemoral & 22 & $\begin{array}{l}\text { Reporte del } \\
\text { paciente }\end{array}$ & $26 \%$ completa \\
\hline Miwa & 10 & 67 & $\begin{array}{l}\text { Canalización abierta } \\
\text { y alcohol }\end{array}$ & 32 & $\begin{array}{l}\text { Reporte del } \\
\text { paciente }\end{array}$ & $50 \%$ completa \\
\hline Peskircioglu & 32 & 46 & $\begin{array}{l}\text { Canalización abierta y } \\
\text { cianoacrilato }\end{array}$ & 25 & $\begin{array}{l}\text { Reporte del } \\
\text { paciente }\end{array}$ & $69 \%$ completa \\
\hline Malossini & 17 & 36 & $\begin{array}{l}\text { Canalización } \\
\text { abierta y coils }\end{array}$ & 34 & $\begin{array}{l}\text { Reporte del } \\
\text { paciente }\end{array}$ & $73 \%$ completa \\
\hline Rebonato & 18 & 51 & $\begin{array}{l}\text { Canalización guiada } x \\
\text { ECO y Cianoacrilato + coil }\end{array}$ & $13.3+/-7$ & IIFE, ECO & NS \\
\hline Hsu GL & 16 & $39-68$ & Venous Stripping & $92.4 \pm 58.8$ & $\begin{array}{l}\text { CAV y } \\
\text { cuestionario }\end{array}$ & $\begin{array}{l}\text { Mejoría del IIEF } \\
\text { en } 13 / 16\end{array}$ \\
\hline Hsu GL & $\begin{array}{l}45 \text { casos vs } \\
38 \text { controles }\end{array}$ & $25-83$ & Venous Stripping & $37.7 \pm 11.5$ & $\begin{array}{l}\text { CAV y } \\
\text { cuestionario }\end{array}$ & No hubo diferencia \\
\hline Hsu GL & $\begin{array}{l}178 \text { casos vs } \\
131 \text { controles }\end{array}$ & $19-81$ & Venous Stripping & $92.4 \pm 16.8$ & $\begin{array}{l}\text { ECO, CAV } \\
\text { y cuestionario }\end{array}$ & $\begin{array}{l}\text { 90,4\% mejoría del } \\
\text { IIEF en casos }\end{array}$ \\
\hline
\end{tabular}

Abreviaturas: CAV, Cavernosografía; DUS, Eco doppler de pene; IIEF, Índice Internacional de función eréctil.

vacilante de la erección (PVE), es decir, con fallas eréctiles ante la disminución de la excitación o concentración deben ser revisados para un diagnostico posible de fuga venosa congénita. El paciente típicamente relata pérdida de la erección al ponerse un condón, cambiar de posición, interrupciones externas como el timbre de un celular, lo que se ha llamado patrón vacilante de la erección (PVE). El llamado síndrome de Fuga venosa dorsal incluye los siguientes componentes potenciales:

- Disfunción eréctil primaria (DEP) o no estándar, antes de los 40 años (DESNE) de más de 6 meses de evolución.

- Patrón vacilante de la erección(PVE): Erecciones blandas, de corta duración o que se caen con facilidad ante perdida del estímulo sexual (Ej: Ponerse un condón, cambios de posición, distracciones externas como el sonido de un celular).

- Los pacientes clásicamente relatan que tienen una falla mayor en posición de pies.

- Erección del 75\% o mayor con el estímulo vasoactivo durante fármaco-ecografía- doppler. Es decir, la fuga no es un artefacto en un pene sin la suficiente erección.
- Criterios ecográficos de fuga venosa: El más importante es la presencia de una fuga venosa dorsal profunda comprobada en el ultrasonido peneano, sin otras fugas venosas en la topografía de los cuerpos cavernosos. (Ver - Figura 1).

\section{Diagnóstico de las Fugas Venosas}

No será suficiente repetir, el número de veces que sea necesario, que el éxito en el tratamiento final de esos pacientes, está basado en la calidad del diagnóstico.

1- Ecografía doppler de pene: La ecografía doppler de pene es una herramienta costo efectiva para la evaluación de la hemodinámica peneana, que permite a su vez dilucidar el estado del sistema arterial y el comportamiento venoso durante las diferentes fases de la erección. Permite encontrar un desequilibrio en el flujo saliente que escapa por la fuga venosa dorsal y altera profundamente la hemodinámica del pene. ${ }^{27-40}$

1- Criterios diagnósticos de enfermedad venoclusiva en ecografía dopler de pene (Ver - Figura 2) 


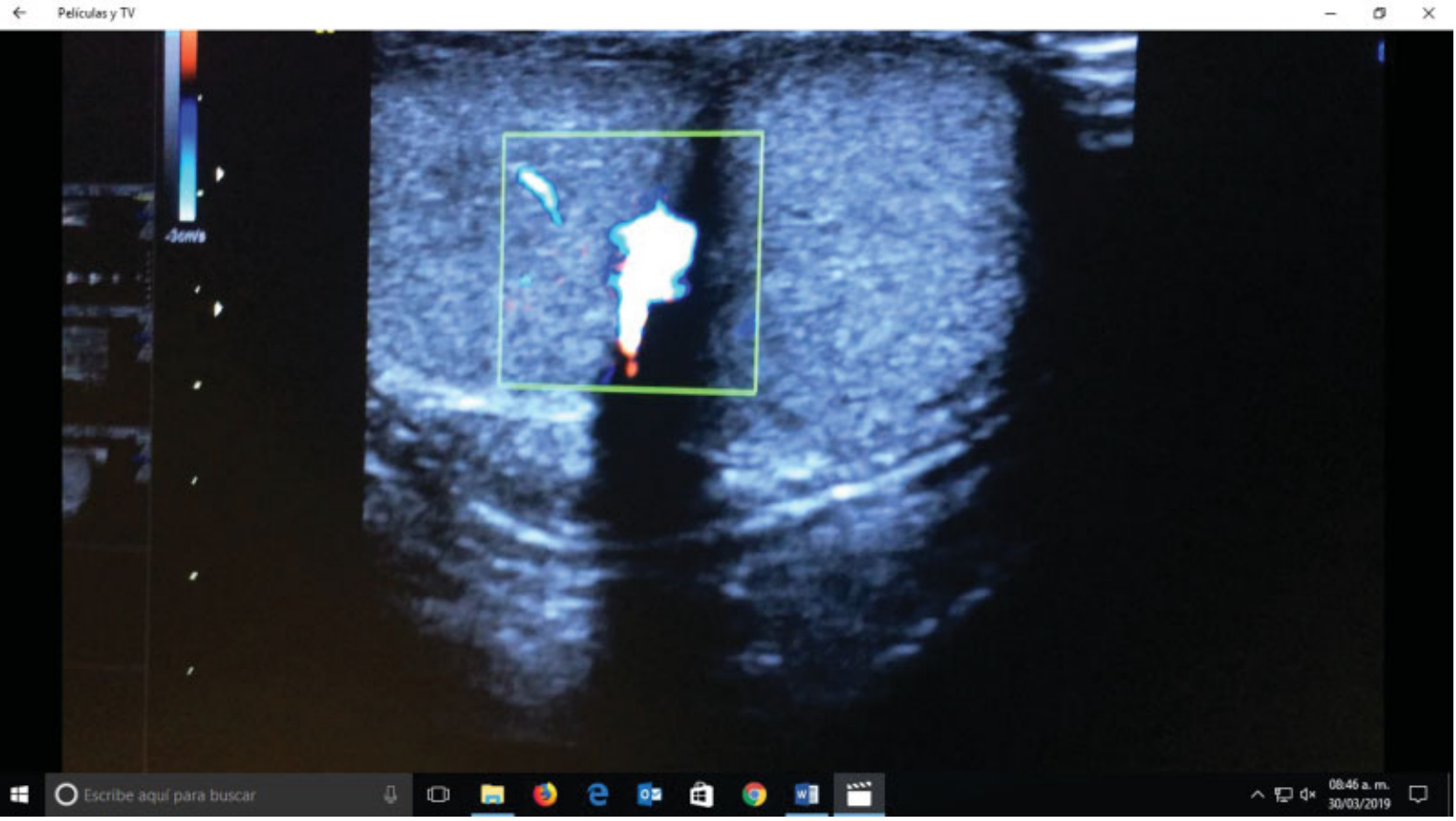

Fig. 1 Fuga venosa dorsal profunda típica (sin registro) en Ecografía dopler de alta definición.

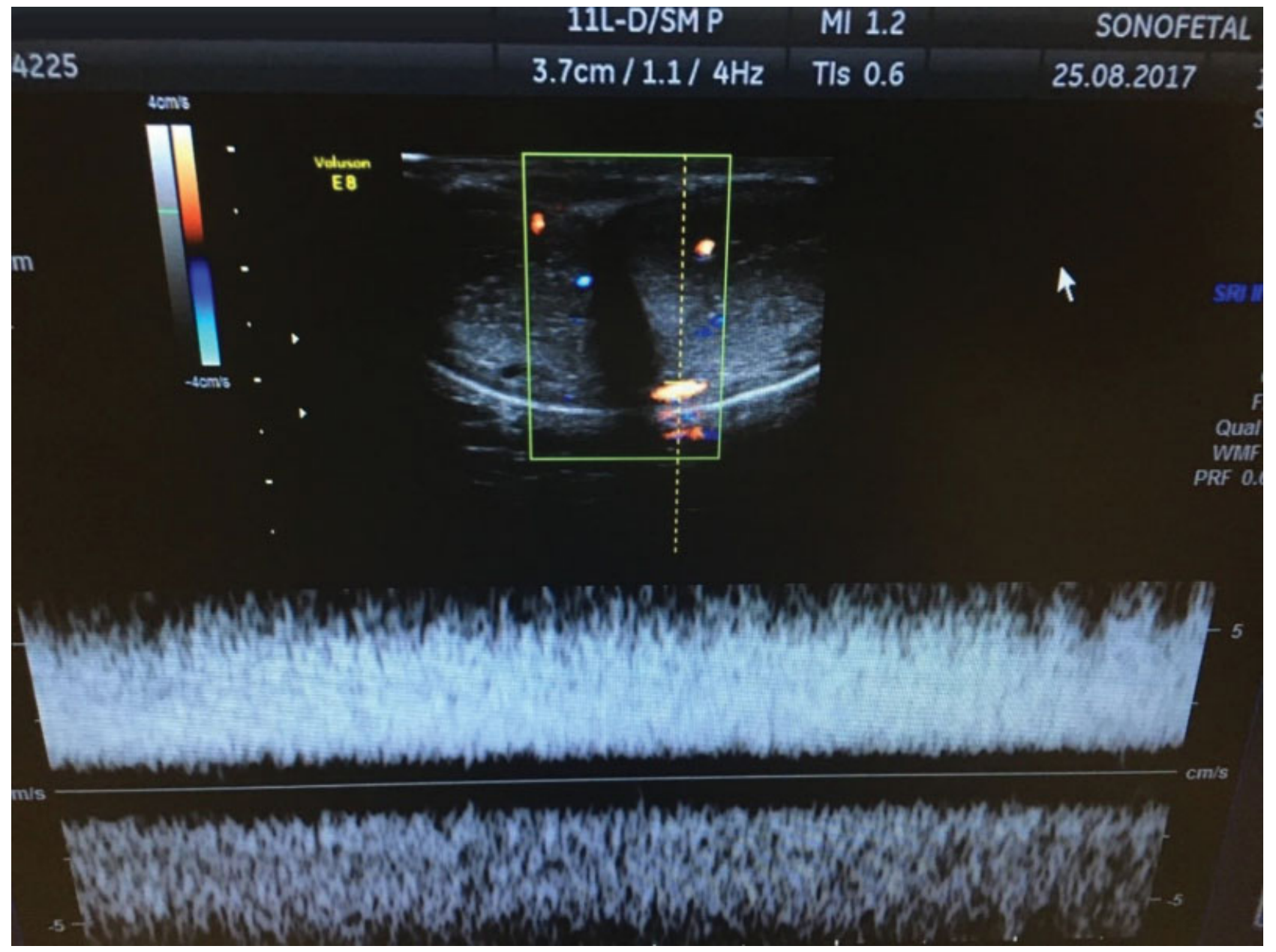

Fig. 2 Fuga venosa dorsal profunda con registro en Ecografía dopler de alta definición. 


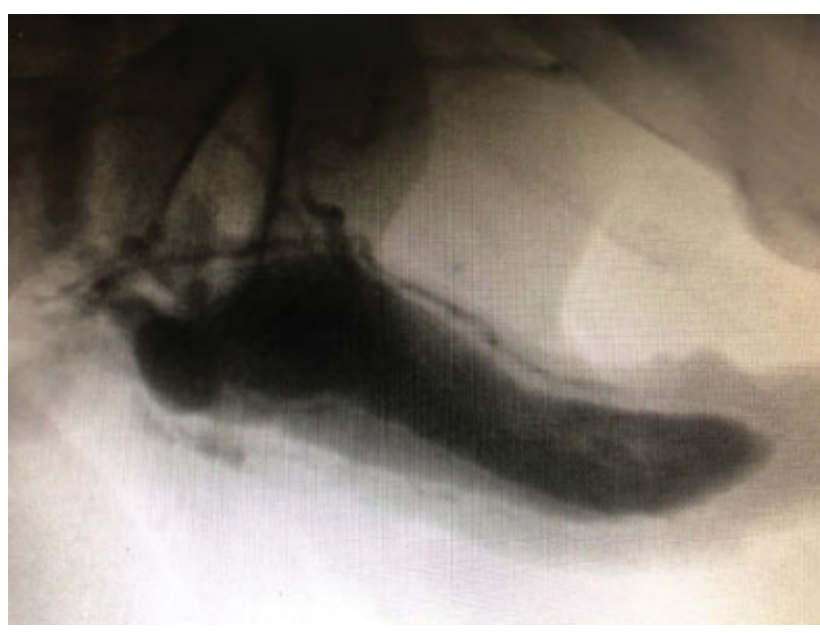

Fig. 3 Fuga venosa dorsal profunda en placa oblicua de una cavernosografía con medio de contraste yodado.

- Velocidad sistólica (PSV) mayor a $30 \mathrm{cc} / \mathrm{seg}$ (Arterias normales).

- Velocidad al final de la diástole (ESV) en 0 o muy cercana.

- Registro de perdida de flujo sanguíneo continuo (fuga), en la vena dorsal profunda del pene durante la totalidad del examen, con tumescencia suficiente del tejido para bloquear el escape venoso.

- No existen otras fugas cavernosas.

- Pueden existir fugas periuretrales (esponjosas) que no afectan la hemodinámica de la erección, o fugas venosas dorsales superficiales que serán tratadas en conjunto.

2- Carvernosografía: Un examen clásico para el diagnóstico de fuga es el uso de un medio de contraste intracavernoso, con placas secuenciales para detectar la fuga venosa $\mathrm{y}$ fotografiarla adecuadamente; es prerrequisito una erección inducida con vasoactivo o al menos con solución salina. El examen idealmente debería estar acompañado de una cavernosometría y en conjunto el examen se denomina en la literatura Dynamic infusion cavernosometry and cavernosography (DICC). Actualmente el examen se realiza en equipos de radiología digital que permitan hacer secuencias en vídeo de la inyección. Es un examen complementario de la ecografía dopler y necesita entrenamiento para ser realizado adecuadamente. (Ver - Figura 3). ${ }^{41,42}$

3- CT Cavernosografía (CavernoTAC) o RMN Cavernosografía (Cavernoresonancia): El uso de otros métodos de imágenes, siempre con uso de contraste intracavernoso (yodados o gadolinio), pueden mejorar notablemente la calidad de las imágenes, con visión tridimensional, rotación, color y reconstrucción de la imagen que incrementan el poder del diagnóstico. (Ver - Figuras 4 y 5)..$^{43,44}$

\section{Opciones de Tratamiento de las Fugas Venosas}

\section{No Quirúrgicas}

1- Uso de terapia oral con IPDE5: El tratamiento con medicamentos vasodilatadores tipo IPDE-5 permite el aumento del flujo entrante del pene, lo que produce una mejoría transitoria de la pérdida de la erección sin mejorar el problema de base. Todos los medicamentos (Sildenafil, Tadalafil, Avanafil y Vardenafil) han sido utilizados, tanto a demanda, o en dosis diaria (tadalafil). El paciente experimenta una mejoría transitoria de su erección, con persistencia del patrón vacilante (PVE) al disminuir la excitación y recaídas en las fallas a medida que envejece.

2- Uso de terapia intracavernosa con vasoactivos: Igual que en la opción anterior, las inyecciones

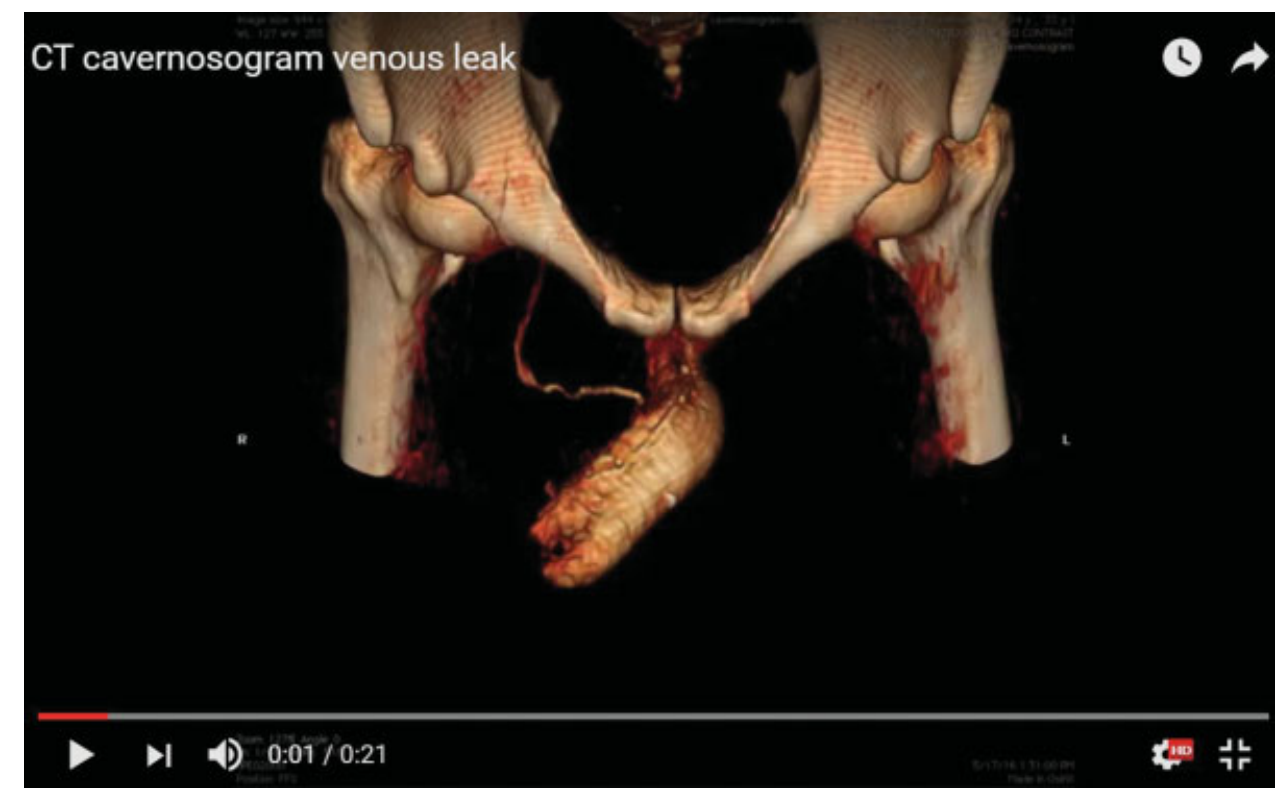

Fig. 4 Fuga venosa dorsal profunda en cavernosografía TAC. Cortesía: Dr. Alejandro Fernández, Clínica de la Mujer - Bogotá. 


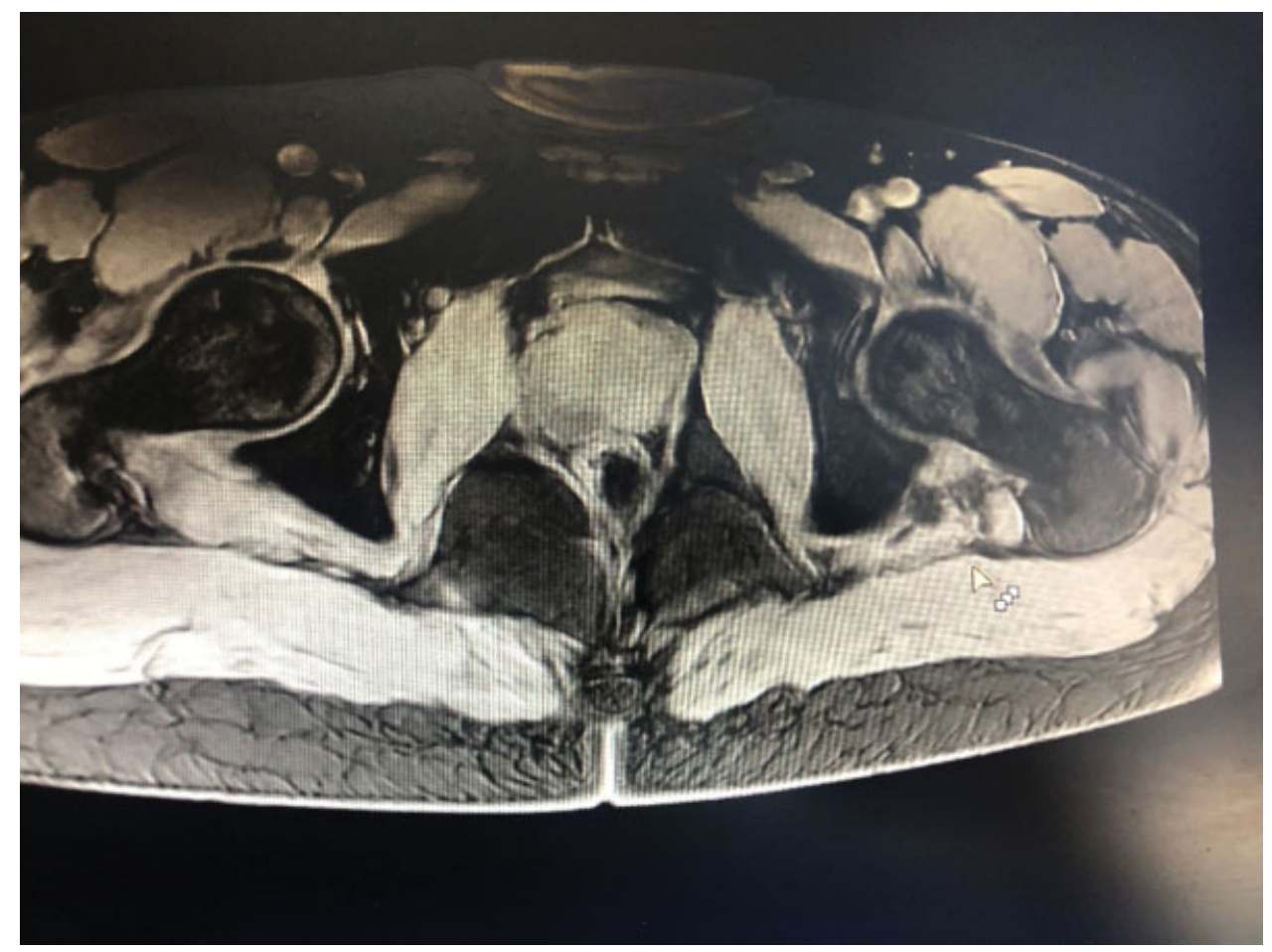

Fig. 5 Resonancia Magnética Nuclear de pene mostrando cruras y ligamento suspensorio.

vasodilatadoras logran cambiar la ecuación entre flujo entrante y flujo saliente. El paciente experimenta una mejoría de la calidad de la erección, utilizando dosis de vasoactivos que a menudo deben ser crecientes, lo que podría incrementar el riesgo de erecciones prolongadas.

3- Uso de anillos controladores de flujo: La oclusión mecánica del flujo en la base del pene puede controlar adecuadamente el problema del flujo saliente. Es un método de tratamiento que necesita adherencia por parte del paciente y su pareja.

4- Aparatos de vacío: Los aparatos de vacío (VED), pueden complementar muy bien la opción anterior y convertirse para quienes tienen adherencia, en un poderoso inductor de erecciones. ${ }^{4}$

5- Uso de ondas de choque de baja intensidad: Algunos estudios muestran resultados prometedores en disfunción eréctil vasculogénica de origen arterial. Por el tipo de mecanismo de acción relacionado con neo vascularización, no se espera una reacción importante cuando el mecanismo subyacente es una fuga venosa, aunque han sido propuestos como una alternativa. ${ }^{45}$

\section{Quirúrgicas}

1- Ligadura de venas dorsales del pene: La cirugía tradicional implica el abordaje de las venas dorsales selectivamente por circuncisión o por una incisión en el cuerpo peneano. La técnica, figura descrita en el apartado final de este artículo en el paso a paso. ${ }^{12,46-50}$

2- Ligadura venosa con técnica venous stripping: En los últimos años, el coreano GL Hsu's ha propuesto técnicas adicionales, incluyendo la denominada venous stripping, la cual es una cirugía más demandante técnicamente, puesto que implica el aislamiento y ligadura selectiva de las venas principales, con búsqueda exhaustiva de las ramas venosas, hasta las más pequeñas, en el cuerpo del pene. ${ }^{51-53}$

3- Ligadura venosa por laparoscopia: Una variación propuesta para la ligadura venosa es el abordaje laparoscópico del plexo de Santorini donde desembocan las venas dorsales del pene. La técnica es semejante a uno de los primeros pasos de hemostasia que se realiza en la prostatectomía radical. Se hace un abordaje del espacio de Retzius y se realiza una ligadura en bloque de dicho plexo pre prostático.

4- Ligadura venosa con uso de aparatos: En proceso de investigación, se encuentra el uso de dispositivos que permiten la implantación de coils de nitinol por un abordaje mínimamente invasivo en el cuerpo del pene.

5- Embolización de venas dorsales: La radiología intervencionista ha propuesto la embolización selectiva de las venas dorsales utilizando agentes que usan productos como el aetoxyesclerol, N-butyl cyanoacrylate, coils endovasculares, o combinaciones de los anteriores. ${ }^{54,55}$

6- Uso de prótesis peneana: Como en otros casos de disfunción eréctil, el uso de prótesis del pene suele ser un recurso final. Debe tenerse en cuenta que esos pacientes a menudo son muy jóvenes y lo que no es aceptable es proponerles un implante sin realizar previamente un estudio adecuado y sin considerar otras opciones de tratamiento. 


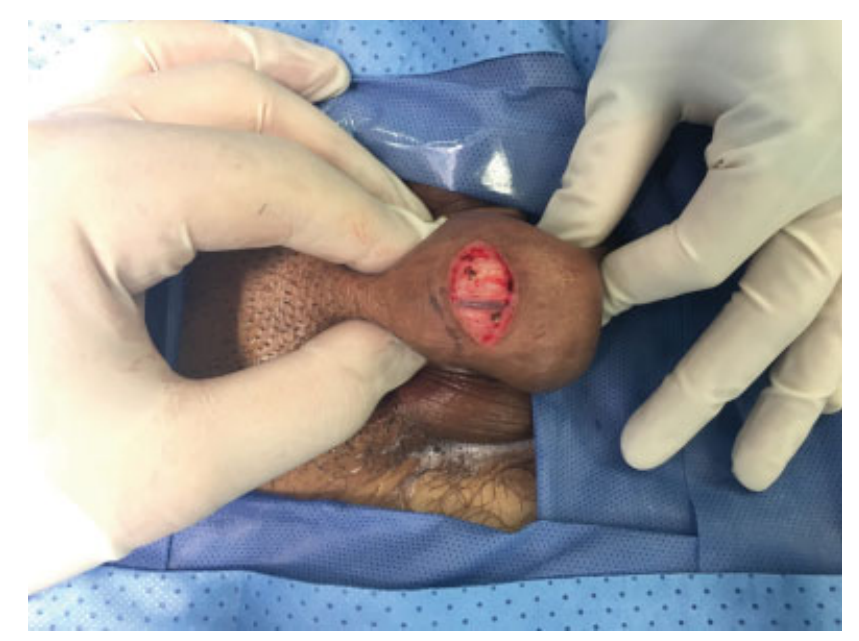

Fig. 6 Fuga venosa dorsal superficial en incisión dorsal del cuerpo peneano.

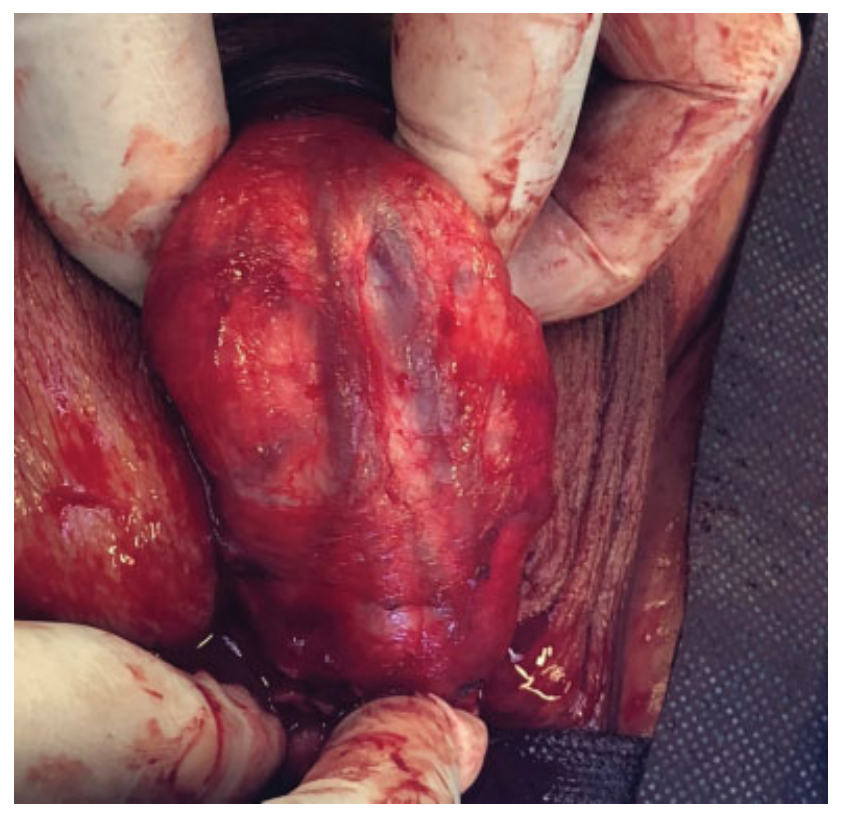

\section{Técnica quirúrgica paso a paso}

1- INCISION (Paso 1): La incisión puede realizarse como una circuncisión clásica o como una incisión dorsal en el cuerpo del pene. Esa segunda opción, limita el campo de visión y la búsqueda de las venas emisarias y perforantes más externas al tronco principal. (Ver - Figura 6).

2- DEGLOVING (Paso 2). El degloving del pene solo puede realizarse durante circuncisión y es lo que le otorga una ventaja adicional a ese método de ingreso, puesto que la visión sobre las venas es superior a la incisión única. (Ver - Figura 7).

3- DISECCIÓN DE LAS VENAS (Paso 3): La vena dorsal superficial típicamente está localizada por debajo de la piel y sobre la fascia superficial del pene o dartos, o fascia de Colles y es fácilmente reconocible con una disección mínima en el dorso del pene; en cambio la vena dorsal profunda, que es la que interesa en esta cirugía, está localizada por debajo de la fascia profunda o de Buck, con las arterias dorsales a su lado y que no necesariamente necesita ser disecada con la técnica que se propone para realizar su ligadura. (Ver - Figuras 8 y 9).

4- CONGESTION PASIVA. (Paso 4): Lo que permite la visualización adecuada de la vena profunda y sus ramas que yacen por debajo de la fascia de Buck, es la realización de una maniobra que permita su llenado y que se denomina "ordeñado" de las venas. Se arquea el cuerpo del pene y de manera gentil se hace presión distal con la yema de los dedos. (Ver - Figura 10).

5- SECCIÓN Y LIGADURA DE LA VENA DORSAL SUPERFICIAL (Paso 5): La sección y ligadura de la vena dorsal facilita la visualización de las venas profundas y se realiza por su facilidad técnica, aunque no es obligatorio. En algunos casos, el panorama de las venas profundas es tan claro que no se requiere la sección, únicamente la ligadura de dicha vena superficial, igual que se hará con las profundas. (Ver - Figura 11).

Fig. 7 Degloving clásico durante la circuncisión.

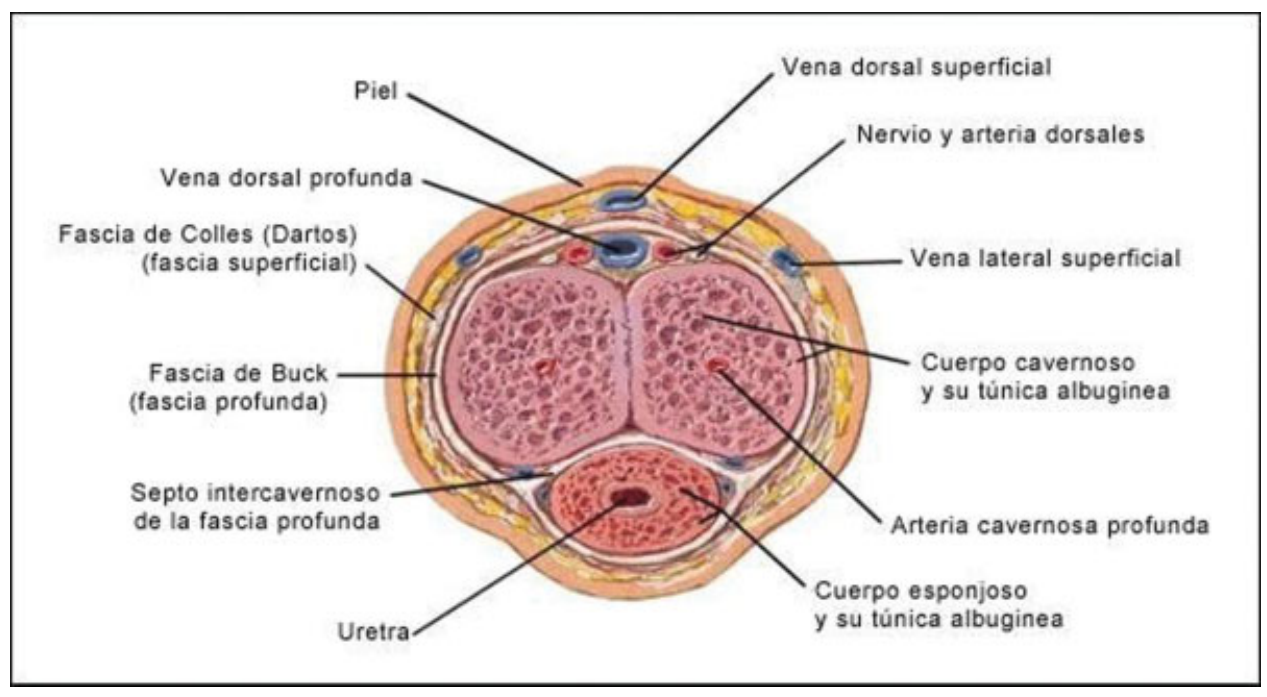

Fig. 8 Esquema de la localización de las venas y las fascias en el pene. 
6- LIGADURA DE LA VENA DORSAL PROFUNDA, VENAS PERFORANTES Y EMISARIAS. (Paso 6): La ligadura se puede realizar in situ, sin hacer una extensa disección de las venas. Idealmente se utiliza magnificación, se hace

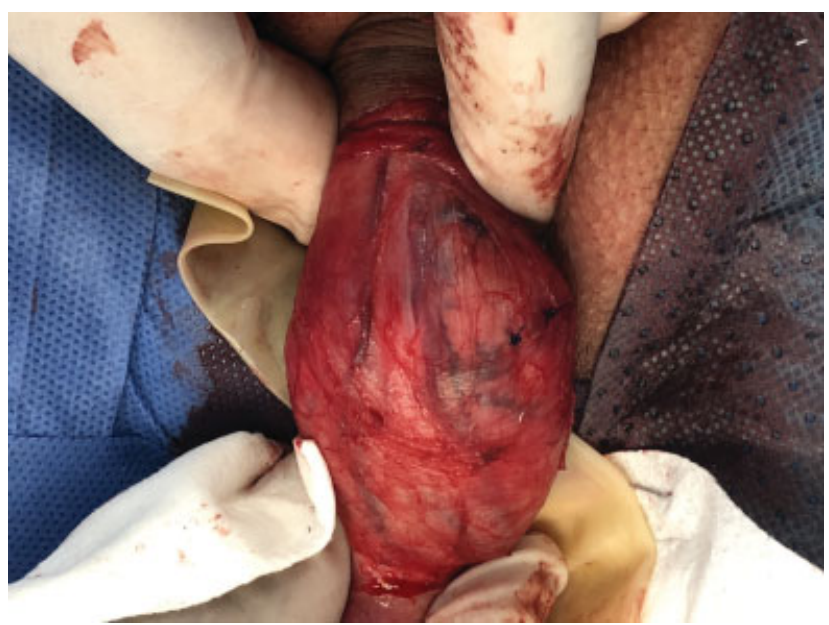

Fig. 9 Venas dorsales superficial y profunda luego de disección de las fascias superficiales del pene.

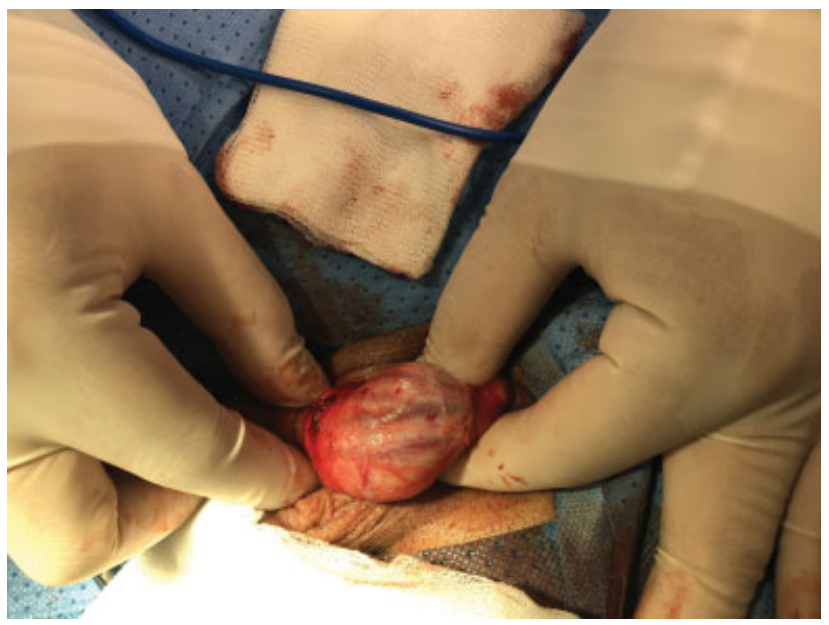

Fig. 10 Congestión pasiva (Ordeñado) de la vena dorsal profunda.

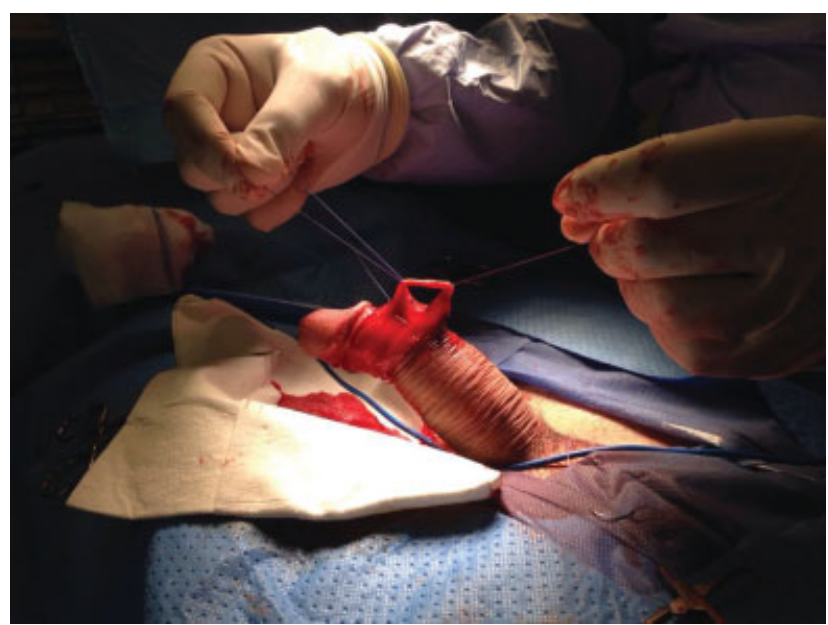

Fig. 11 Vena dorsal superficial aislada para seccionar y ligar. previamente la maniobra de ordenamiento, se despeja la vena dorsal superficial y para la ligadura se utiliza una sutura absorbible tipo PDS o monocryl 4-0. Las venas deben abordarse desde las más periféricas, menos gruesas, hacia el centro donde estarán las más voluminosas. (Ver - Figuras 12 y 13).

7- FINAL DE LA CIRUGíA. (Paso 7): La piel se cierra como habitualmente en una circuncisión y en el caso de incisiones corporales se sugiere el uso de dos planos para evitar disrupciones por las erecciones nocturnas que suelen aumentar notablemente después de esa cirugía. El paciente debería tener un bloqueo anestésico tipo bupivacaína al final de la cirugía. Aunque el riesgo de hematoma es mínimo, es deseable el uso de un vendaje de

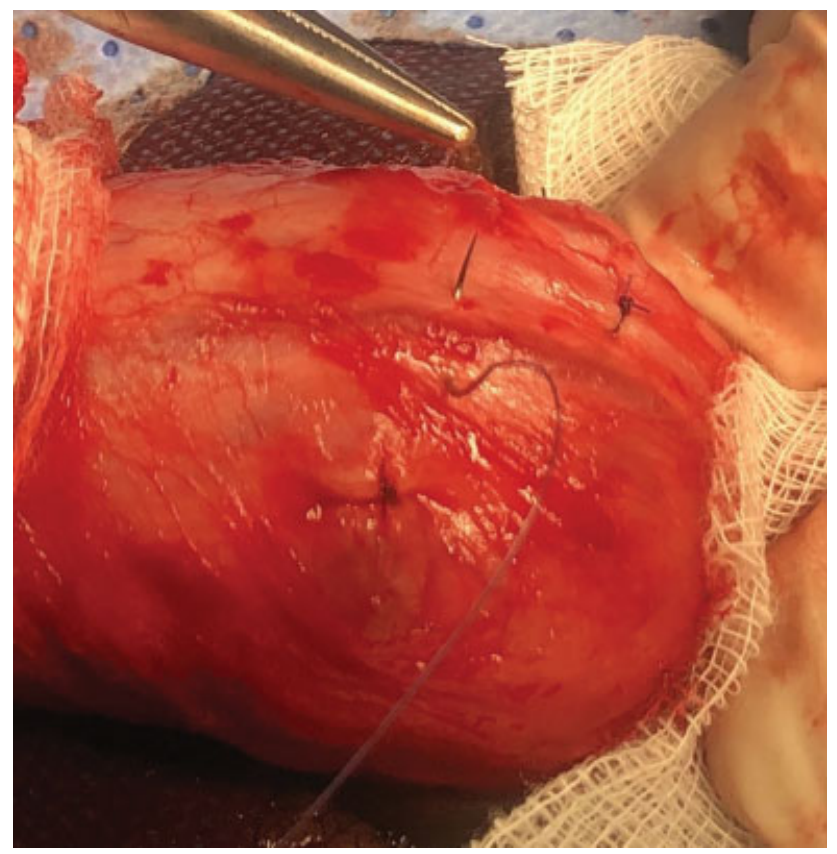

Fig. 12 Ligadura selectiva de vena dorsal profunda, emisarias y perforantes.

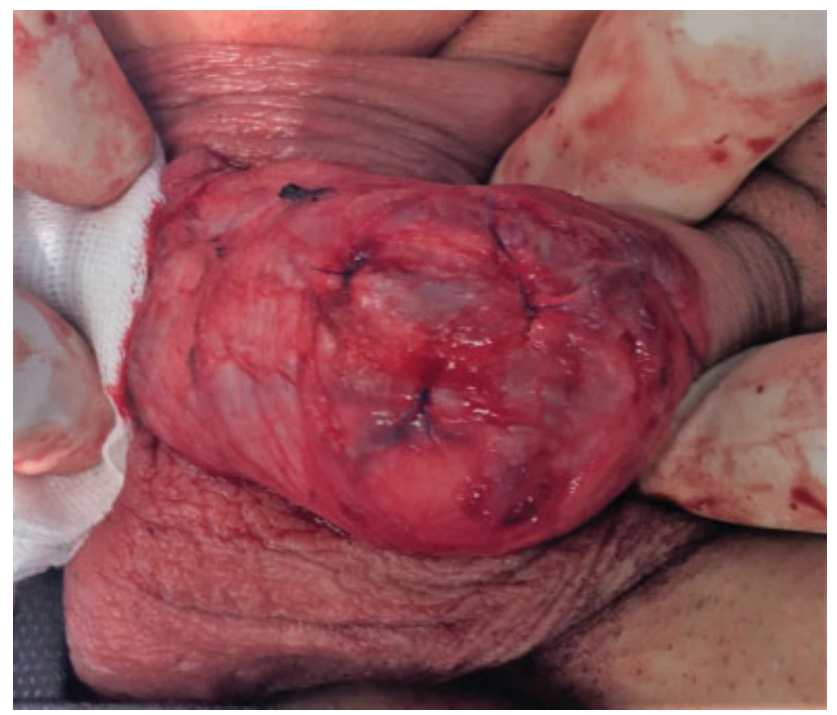

Fig. 13 Ligadura selectiva de vena dorsal profunda, emisarias y perforantes. 


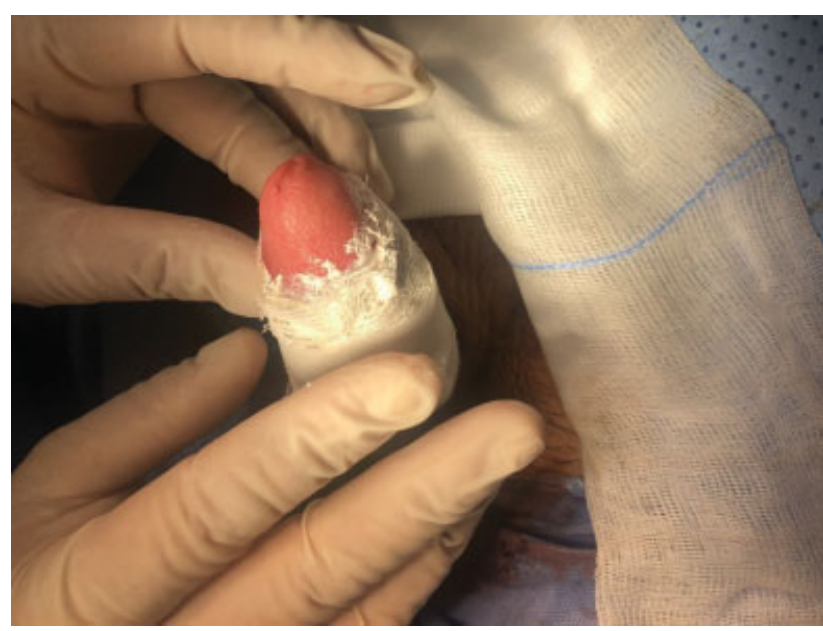

Fig. 14 Vendaje final con óxido de zinc.

tela, untado de crema tipo óxido de zinc o furacin, que permita el uso cómodo de hielo durante las primeras 24 horas. (Ver - Figura 14).

\section{Conclusiones}

Cuando se sospecha la posibilidad diagnóstica de una fuga venosa dorsal profunda con alteraciones hemodinámicas que expliquen un cierto tipo de disfunción eréctil, se configura una entidad definida como Síndrome de fuga vena dorsal profunda, con unos hallazgos clínicos dominados por un patrón vacilante de la erección (PVE), y unos hallazgos ecográficos que incluyen un estado arterial normal y un registro persistente de fuga por la vena dorsal profunda del pene, que podrían ser corroborados con los otros métodos diagnósticos disponibles. Aunque existen algunas opciones médicas de tratamiento, se propone la corrección quirúrgica de la fuga venosa como el método ideal de tratamiento definitivo. Otras fugas pueden ser encontradas durante la exploración, pero su manejo quirúrgico tiene escaso o nulo soporte en la literatura. Por lo general, en ese tema, son necesarios más estudios y en el futuro se esperan nuevos desarrollos con respecto a la génesis de fugas venosas congénitas.

Conflicto de Intereses

Los autores declaramos que no existen conflictos de intereses.

\section{Referencias}

1 Lue TF. Physiology of Penile Erection and Pathophysiology of Erectile Dysfunction. En: Alan J. Wein; editors, Louis R. Kavoussi, Alan W. Partin, Craig A. Peters. Campbell-Walsh Urology. Vol 1.11va ed. Philadelphia: Elsevier; 2016:612-642

2 Shafik A, Shafik I, El Sibai O, Shafik AA. On the pathogenesis of penile venous leakage: role of the tunica albuginea. BMC Urol 2007;7:14

3 Wooten JS. Ligation of the dorsal vein of the penis as a cure for atonic impotence. Texas Med J. 1902;18:325-328

4 Wespes E, Schulman C. Venous impotence: pathophysiology, diagnosis and treatment. JUrol 1993;149(5 Pt 2):1238-1245
5 Wespes E, Raviv G, Vanegas JP, et al. Corporeal veno-occlusive dysfunction: a distal arterial pathology? JUrol 1998;160(6 Pt 1):2054-2057

6 Lazzarini H, de Miseu S, Lopez S, Irazu J. Síndrome de escape venoso: Causa de disfunción sexual masculina. Rev Argent Urol 1990;55(03): $\cdots$

7 Shabsigh R, Fishman IJ, Toombs BD, Skolkin M. Venous leaks: anatomical and physiological observations. JUrol 1991;146(05): 1260-1265

8 Lue TF, Hricak H, Marich KW, Tanagho EA. Vasculogenic impotence evaluated by high-resolution ultrasonography and pulsed Doppler spectrum analysis. Radiology 1985;155(03):777-781

9 Flores S, Tal R, O'Brien K, Mulhall JP. Outcomes of crural ligation surgery for isolated crural venous leak. JSex Med 2011;8(12): 3495-3499

10 Cayan S. Primary penile venous leakage surgery with crural ligation in men with erectile dysfunction. JUrol 2008;180(03): 1056-1059

11 Cakan M, Yalçinkaya F, Demirel F, Ozgünay T, Altuğ U. Is dorsale penile vein ligation (DPVL) still a treatment option in venoocclusive dysfunction? Int Urol Nephrol 2003;35(04):529-534

12 Schultheiss D, Truss MC, Becker AJ, Stief CG, Jonas U. Long-term results following dorsal penile vein ligation in 126 patients with veno-occlusive dysfunction. Int J Impot Res 1997;9(04):205-209

13 Lukkarinen O, Tonttila P, Hellström P, Leinonen S. Non-prosthetic surgery in the treatment of erectile dysfunction. A retrospective study of 45 impotent patients in the University of Oulu. Scand J Urol Nephrol 1998;32(01):42-46

14 Basar MM, Atan A, Yildiz M. Long-term results of venous ligation in patients with veno-occlusive dysfunction. Int J Impot Res 1998; 10S:21

15 Al Assal F, Delgado A, Al Assal R. Venous surgery for venoocclusive dysfunction. Long-term results. Int J Impot Res 1998;10S:31

16 Sasso F, Gulino G, Weir J, Viggiano AM, Alcini E. Patient selection criteria in the surgical treatment of veno-occlusive dysfunction. J Urol 1999;161(04):1145-1147

17 Popken G, Katzenwadel A, Wetterauer U. Long-term results of dorsal penile vein ligation for symptomatic treatment of erectile dysfunction. Andrologia 1999;31(Suppl 1):77-82

18 Rao DS, Donatucci CF. Vasculogenic impotence. Arterial and venous surgery. Urol Clin North Am 2001;28(02):309-319

19 Trost LW, Munarriz R, Wang R, Morey A, Levine L. External Mechanical Devices and Vascular Surgery for Erectile Dysfunction. J Sex Med 2016;13(11):1579-1617. Doi: 10.1016/j. jsxm.2016.09.008

20 Hsu GL, Hung YP, Tsai MH, et al. Penile veins are the principal component in erectile rigidity: a study of penile venous stripping on defrosted human cadavers. J Androl 2012;33(06):1176-1185

21 Hsu GL, Hsieh CH, Wen HS, Kang TJ, Chiang HS. Penile venous anatomy: application to surgery for erectile disturbance. Asian J Androl 2002;4(01):61-66

$22 \mathrm{Hsu}$ GL, Hsieh CH, Wen HS, et al. Anatomy of the human penis: the relationship of the architecture between skeletal and smooth muscles. J Androl 2004c25(03):426-431

23 Uribe JF. Parámetros hemodinámicos en el proceso normal de la erección. Urol Colomb. 2015;24(02):101-105

24 Tal R, Valenzuela R, Aviv N, et al. Persistent erectile dysfunction following radical prostatectomy: the association between nervesparing status and the prevalence and chronology of venous leak. J Sex Med 2009;6(10):2813-2819

25 Broderick G. Valoración vascular de la disfunción eréctil. En: Lue Tom. Atlas de la disfunción sexual masculina. San Francisco: Current Medical Group; 2008

26 Burnett AL, Nehra A, Breau RH, et al. Erectile Dysfunction: AUA Guideline. JUrol 2018;200(03):633-641. Doi: 10.1016/j.juro.2018. 05.004

27 Chen L, Xu L, Wang J, et al. Diagnostic Accuracy of Different Criteria of Pharmaco-penile Duplex Sonography for Venous Erectile 
Dysfunction. JUltrasound Med 2019;38(10):2739-2748. Doi: 10.1002/jum.14982

28 Papagiannopoulos D, Khare N, Nehra A. Evaluation of young men with organic erectile dysfunction. Asian J Androl 2015;17(01): 11-16

29 Lue TF, Mueller SC, Jow YR, Hwang TI. Functional evaluation of penile arteries with duplex ultrasound in vasodilator-induced erection. Urol Clin North Am 1989;16(04):799-807

30 Teloken PE, Park K, Parker M, Guhring P, Narus J, Mulhall JP. The false diagnosis of venous leak: prevalence and predictors. J Sex Med 2011;8(08):2344-2349

31 Berookhim BM. Doppler Duplex Ultrasonography of the Penis. J Sex Med 2016;13(04):726-731

32 Roy C, Saussine C, Tuchmann C, Castel E, Lang H, Jacqmin D. Duplex Doppler sonography of the flaccid penis: potential role in the evaluation of impotence. JClin Ultrasound 2000;28(06): 290-294

33 Speel TG, van Langen H, Wijkstra H, Meuleman EJ. Penile duplex pharmaco-ultrasonography revisited: revalidation of the parameters of the cavernous arterial response. JUrol 2003;169(01):216-220

34 Mills RD, Sethia KK. Reproducibility of penile arterial colour duplex ultrasonography. Br J Urol 1996;78(01):109-112

35 Gilbert BR, Paduch D. Penile Dopler Ultrasound. Post graduate course, AUA Annual Meeting, Orlando Fla. May 17, 2014.

36 Aversa A, Sarteschi LM. The role of penile color-duplex ultrasound for the evaluation of erectile dysfunction. J Sex Med 2007;4(05): 1437-1447

37 Sarteschi LM, Palego P, Lencioni R. Eco-colorDoppler peniena dinamica: Quadro normale. In: Sarteschi LM, Menchini Fabris GF, eds. Ecografia andrologica. Modena, Italy: Athena Audiovisuals; 2003:173-184

38 Wilkins CJ, Sriprasad S, Sidhu PS. Colour Doppler ultrasound of the penis. Clin Radiol 2003;58(07):514-523

39 Mancini M, Bartolini M, Maggi M, Innocenti P, Villari N, Forti G. Duplex ultrasound evaluation of cavernosal peak systolic velocity and waveform acceleration in the penile flaccid state: clinical significance in the assessment of the arterial supply in patients with erectile dysfunction. Int J Androl 2000;23(04):199-204

40 Schaeffer EM, Jarow JPJr, Vrablic J, Jarow JP. Duplex ultrasonography detects clinically significant anomalies of penile arterial vasculature affecting surgical approach to penile straightening. Urology 2006; 67(01):166-169

41 Hsu GL, Hill JW, Chen HS, Huang SJ. Novel pilot films providing indispensable information in pharmaco-cavernosography. Transl Androl Urol 2015;4(04):398-405

42 Gao QQ Chen JH, Chen Y, Song T, Dai YT. Dynamic infusion cavernosometry and cavernosography for classifying venous erectile dysfunction and its significance for individual treatment.
Chin Med J (Engl) 2019;132(04):405-410. Doi: 10.1097/CM9.0000 000000000099

43 Ye T, Li J, Li L, Yang L. Computed tomography cavernosography combined with volume rendering to observe venous leakage in young patients with erectile dysfunction. Br J Radiol 2018;91 (1091):20180118

44 Xu CC, Ruan XZ, Tang YF, Pan JH, Wang GY, Huang QL. Diagnostic value of four-dimensional CT angiography in arterial erectile dysfunction using 320-detector row dynamic volume CT. Biosci Rep 2017;37(04):x

45 Brunckhorst O, Wells L, Teeling F, Muir G, Muneer A, Ahmed K. A systematic review of the long-term efficacy of low-intensity shockwave therapy for vasculogenic erectile dysfunction. Int Urol Nephrol 2019;51(05):773-781

46 Berardinucci D, Morales A, Heaton JP, Fenemore J, Bloom S. Surgical treatment of penile veno-occlusive dysfunction: is it justified? Urology 1996;47(01):88-92

47 Da Ros CT, Telöken C, Antonini CC, Sogari PR, Souto CA. Long-term results of penile vein ligation for erectile dysfunction due to cavernovenous disease. Tech Urol 2000;6(03):172-174

48 Kim ED, McVary KT. Long-term results with penile vein ligation for venogenic impotence. JUrol 1995;153(3 Pt 1):655-658

49 Knigavko O, Lesovoy V, Arkatov A, et al. Correction of venoocclusive form of erectile dysfunction. JSex Med 2015;12 (Suppl 3):241-271

50 Sohn M, Hatzinger M, Goldstein I, Krishnamurti S. Standard operating procedures for vascular surgery in erectile dysfunction: revascularization and venous procedures. JSex Med 2013;10(01): 172-179

51 Hsu GL, Chen HS, Hsieh CH, Lee WY, Chen KL, Chang CH. Salvaging penile venous stripping surgery. J Androl 2010;31(03):250-260. Doi: $10.2164 /$ jandrol.109.008409

52 Hsu GL, Chen HS, Hsieh CH, et al. Insufficient response to venous stripping surgery: is the penile vein recurrent or residual? J Androl 2006;27(05):700-706

53 Hsu GL, Chen HS, Hsieh CH, Lee WY, Chen KL, Chang CH. Clinical experience of a refined penile venous stripping surgery procedure for patients with erectile dysfunction: is it a viable option? JAndrol 2010;31(03):271-280. Doi: 10.2164/jandrol. 109.008532

54 Rebonato A, Auci A, Sanguinetti F, et al. Embolization of the periprostatic venous plexus for erectile dysfunction resulting from venous leakage. JVasc Interv Radiol 2014;25(06):866-872. Doi: 10.1016/j.jvir.2014.01.015

55 Herwig R, Sansalone S. Venous leakage treatment revisited: pelvic venoablation using aethoxysclerol under air block technique and Valsalva maneuver. Arch Ital Urol Androl 2015;87(01):1-4. Doi: 10.4081/aiua.2015.1.1 